\title{
The p53 inducing drug dosage may determine quiescence or senescence
}

\author{
David P. Lane ${ }^{1}$, Chandra Verma ${ }^{2}$, and Cheok Chit Fang ${ }^{1}$ \\ ${ }^{1}$ p53 lab (A*STAR), \#6-06 Immunos Singapore 138648 \\ ${ }^{2}$ Bionformatics Institute (A*STAR), 30 Biopolis Street, 07-01 Matrix, Singapore 138671
}

Commentary on: Leontieva OV et al. Weak p53 permits senescence during cell cycle arrest. Cell Cycle. 2010; 9:4323-4327.

E-mail: dplane@p53lab.a-star.edu.sg

The protein $\mathrm{p} 53$ regulates the fate of cells [1]. Blagosklonny and his colleagues [2], and others [3] in a series of studies have been using the non-genotoxic p53 activator, Nutlin-3a, to understand further how p53 induction can determine whether cells arrest die or senesce. In earlier work the p53 dependence of cell cycle arrest, apoptosis, autophagy and senescence has been un-equivocally demonstrated both in whole animal and tissue culture models. In these studies however p53 induction was usually achieved by treatment with a DNA damaging drug or ionizing radiation and the question arises as to how much of the cell fate decision is mediated directly by p53 induction and how much by the fact that p53 is being induced in the environment of a "damaged" cell. Indeed when genetic "reactivation " of p53 is induced in lymphoma models [4] apoptosis is the mechanism of tumor elimination, while in a liver tumor model, p53 induced senescence and macrophage engulfment is the route to regression [5]. In these models however the cells are tumor cells, so p53 induction is still taking place in a "damaged or stressed" cell environment. Now using Nutlin-3a alone at varying doses in normal cells Leontieva et al [6] showed that the response to p53 is very sensitive indeed to the level and duration of the $\mathrm{p} 53$ response. This seems to be due to the ability of high levels of p53 to induce both cell cycle arrest and inhibition of the mTOR pathway, while at lower doses, p21 is induced but mTOR inhibition is not. Simply put the inhibition of the cell cycle without mTOR inhibition leads to senescence while dual inhibition leads to a reversible quiescence. This can then lead to the counter-intuitive observation that cells recover better from high doses than from low doses of Nutlin-3a In addition holding cells in a quiescent state for too long helps to promote senescence so both the dose and duration of the p53 signal are critical for outcome. These studies are beginning to throw important light on critical issues of the response of both normal and tumour tissue to therapy. Indeed Leontieva et al showed that Doxorubicin used at the wrong dose/duration might induce a reversible quiescence rather than the desired irreversible senescence or apoptosis in p53 wild type tumor cells. This could help to explain earlier observations by de The and group [7,8] that women with advanced breast cancer show a better response to DNA damaging chemotherapy when they have mutant rather than wild type $\mathrm{p} 53$.

\section{REFERENCES}

1. Vogelstein B et al. Surfing the p53 network. Nature. 2000; 408:307-310.

2. Korotchkina LG et al. The choice between p53-induced senescence and quiescence is determined in part by the mTOR pathway. Aging. 2010; 2:344-352.

3. Huang $B$ et al. Pharmacologic p53 activation blocks cell cycle progression but fails to induce senescence in epithelial cancer cells. Mol Cancer Res. 2009; 7:1497-3509.

4. Ventura $A$ et al. Restoration of p53 function leads to tumour regression in vivo. Nature. 2007; 445:661-665.

5. Xue $W$ et al. Senescence and tumour clearance is triggered by p53 restoration in murine liver carcinomas. Nature. 2007; 445:656-660.

6. Leontieva OV et al. Weak p53 permits senescence during cell cycle arrest. Cell Cycle. 2010; 9: 4323-4327.

7. Bertheau $P$ et al. Exquisite sensitivity of TP53 mutant and basal breast cancers to a dose-dense epirubicin-cyclophosphamide regimen. PLoS Med. 2007; 4:e90.

8. Varna M et al. Int J Cancer. 2009; 124:991-997. 